\title{
Caractérisation hydrogéochimique des eaux des aquifères fissurés de la zone Guiglo-Duekoué (Ouest de la Côte d'Ivoire)
}

\author{
Michel Amani KOUASSI ${ }^{1 *}$, Ernest Kouassi AHOUSSI ${ }^{2}$, Blaise Yao KOFFI ${ }^{2}$, \\ Yves Angbo $\mathrm{AKE}^{3}$ et Jean BIEMI ${ }^{2}$ \\ ${ }^{l}$ Institut National Polytechnique Félix Houphouët-Boigny (INP-HB), Département des Sciences de la Terre et \\ des Ressources Minières (STeRMi) ; BP 1093 Yamoussoukro (Côte d'Ivoire), \\ Tél.(+225) 30646715 ; E-mail : michel.a_kouassi@yahoo.fr. \\ ${ }^{2}$ Université de Cocody-Abidjan, Unité de Formation et de Recherche (UFR) des Sciences de la terre et des \\ Ressources Minières (STRM) ; 22 BP 582 Abidjan 22 ; Tel : (+225) 22483803 (Côte d'Ivoire) ; \\ E-mail : ahoussi@gmx.fr \\ ${ }^{3}$ Ecole de Formation Continue et de Perfectionnement des Cadres (EFCPC), Institut National Polytechnique \\ Félix Houphouët Boigny (INP-HB) ; BP 1093 Yamoussoukro (Côte d'Ivoire), \\ Tél. (+225) 04755542 ; E-mail : akesnell@yahoo.fr. \\ *Auteur correspondant, E-mail: michel.a_kouassi@yahoo.fr
}

\section{RESUME}

La zone d'étude, Guiglo-Duekoué, est située à l'Ouest de la Côte d'Ivoire, entre les latitudes $7^{\circ} 00^{\prime}$ et $7^{\circ} 42^{\prime}$ Nord et les longitudes $6^{\circ} 28^{\prime}$ et $6^{\circ} 50^{\prime}$ Ouest. Cette étude vise à faire une analyse hydrogéochimique les eaux souterraines de la région de Guiglo-Duekoué. L'approche méthodologique est basée sur la détermination des hydrofaciès à partir des diagrammes de Piper et de Schoeller-Berkaloff et l'étude de la qualité des eaux qui s'est appuyée sur une analyse comparative des teneurs des paramètres physico-chimiques aux normes OMS. Les résultats de l'étude ont montré que les eaux sont bicarbonatées calciques et magnésiennes $(95,83 \%)$ et bicarbonatées sodi-potassiques $(4,17 \%)$. Ces eaux ont des températures qui varient entre $25,7{ }^{\circ} \mathrm{C}$ et $28,9{ }^{\circ} \mathrm{C}$, avec une moyenne de $26,7^{\circ} \mathrm{C}$. Leurs $\mathrm{pH}$ oscillent entre 6,51 et 7,13 , avec une moyenne de 6,67 . La turbidité des eaux souterraines reste très faible avec des valeurs qui varient entre $0,20 \mathrm{NTU}$ et 1,01 NTU, pour une moyenne de 0,49 NTU. La conductivité électrique des eaux varie entre $106 \mu \mathrm{S} / \mathrm{cm}$ et $349 \mu \mathrm{S} / \mathrm{cm}$, avec une moyenne de 208,437 $\mu \mathrm{S} / \mathrm{cm}$. La majorité des échantillons d'eau a une conductivité électrique comprise entre $100 \mu \mathrm{s} / \mathrm{cm}$ et $250 \mu \mathrm{s} / \mathrm{cm}(68,75 \%)$. La dureté des eaux est comprise entre $2,8{ }^{\circ} \mathrm{F}$ et $15,40{ }^{\circ} \mathrm{F}$, avec une moyenne de $6,41^{\circ} \mathrm{F}$. La qualité physico-chimique des eaux souterraines étudiées est conforme dans l'ensemble aux normes recommandées par l'OMS et ne présentent pas de danger majeur pour la consommation humaine. Cependant, il existe de fortes teneurs en fer $(0,66 \mathrm{mg} / \mathrm{L})$ et en manganèse $(0,16 \mathrm{mg} / \mathrm{L})$, ce qui explique l'apparence rougeâtre et le goût désagréable de ces eaux.

(C) 2012 International Formulae Group. All rights reserved.

Mots clés: Eaux souterraines; Diagrammes d'analyses hydrochimiques; Normes de l'OMS ; faciès hydrochimiques ; Potabilité ; Région ouest semi-montagneuse. 


\section{INTRODUCTION}

La région Ouest de la Côte d'Ivoire, devenue la nouvelle boucle du cacao, connaît depuis quelques décennies un important essor économique avec un grand développement des activités agricoles associé à une forte expansion démographique (Brou, 2005). Par conséquent, les eaux souterraines dans la région sont de plus en plus utilisées par les populations en général et particulièrement par le monde rural pour leurs besoins domestiques. Mais, très souvent ces eaux sont d'une coloration rougeâtre et ont un goût désagréable, d'où la nécessité une bonne connaissance de la qualité de ces eaux. En effet, la qualité d'une eau souterraine dépend de la nature géologique du sol d'où elle provient et aussi des substances réactives qu'elle aurait pu rencontrer lors de l'écoulement. Ainsi la composition quantitative et qualitative de l'eau souterraine en matières en suspension et dissoutes, de nature minérale ou organique, détermine sa qualité. Cependant cette qualité peut être par exemple altérée lorsque certains éléments chimiques sont présents en des concentrations excessives.

Ainsi, l'objectif de ce travail est de contribuer à une analyse hydrogéochimique des eaux souterraines de la région ouest semimontagneuse. Ce travail a été mené à partir des résultats d'analyses de paramètres physico-chimiques des eaux. La méthodologie appliquée est basée sur la détermination des hydrofaciès et l'analyse comparative des teneurs des paramètres physico-chimiques aux normes OMS (2006). Cette démarche méthodologique a permis d'apporter des réponses à notre problématique.

\section{MATERIEL ET METHODES}

Présentation de la zone d'étude

La zone Guiglo-Duekoué est située à l'Ouest de la Côte d'Ivoire et localisée entre les latitudes $7^{\circ} 00$ et $7^{\circ} 42$ Nord et les longitudes $6^{\circ} 28$ et $6^{\circ} 50$ Ouest (Figure 1). La Côte d'Ivoire est située en Afrique de l'Ouest, dans la zone intertropicale, entre l'équateur et le tropique du cancer, précisément entre les latitudes $4^{\circ} 30^{\prime}$ et $10^{\circ} 30^{\prime}$ Nord et les longitudes $8^{\circ} 30$ et $2^{\circ} 30$ Ouest. Ce pays couvre une superficie de $322462 \mathrm{Km}^{2}$ et fait frontière avec le Golf de Guinée au Sud, le Ghana à l'Est, le Libéria et la Guinée à l'Ouest, le Mali et le Burkina-Faso au Nord.

Le régime climatique qui règne dans la zone Guiglo-Duekoué est un climat subéquatorial de type montagneux. Il est caractérisé par des précipitations annuelles qui varient entre $1600 \mathrm{~mm}$ et $2000 \mathrm{~mm}$. La température moyenne oscille autour de $25^{\circ} \mathrm{C}$ par an. La végétation est constituée de forêts denses humides marquées par endroits de prairies (Brou, 2005). Cette végétation est soumise à l'action anthropique, car l'Ouest de la Côte d'Ivoire est devenu aujourd'hui la nouvelle «boucle du cacao» (Brou, 2005). Cette région est assez accidentée avec une altitude moyenne de $300 \mathrm{~m}$. On y rencontre de vastes et hauts plateaux, de plaines, de basfonds très étendus et de chaînes de montagnes. Les sols sont de type ferralitique à fertilité moyenne et constituent un large domaine pour le développement de l'agriculture, ce qui justifie le déplacement de la boucle du cacao dans cette zone. Il existe également des sols développés sur des roches basiques potentiellement riches en sels minéraux et des sols hydromorphes situés dans les bas-fonds. La couverture hydrographique de la région Guiglo-Duékoué est assurée par le fleuve Sassandra et ses nombreux affluents. 


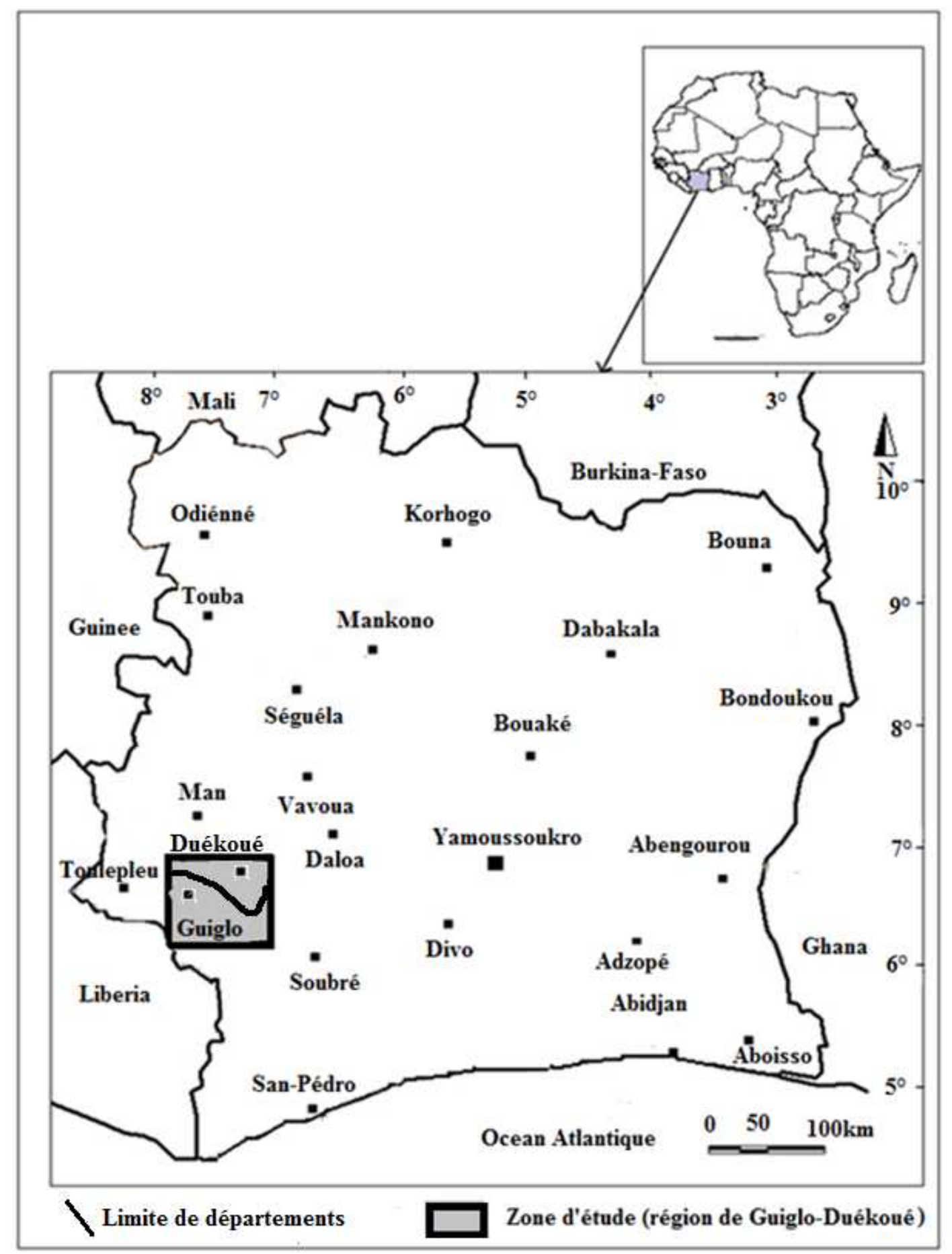

Figure 1 : Localisation de la zone Guiglo-Duekoué (Ouest de la Côte d'Ivoire). 


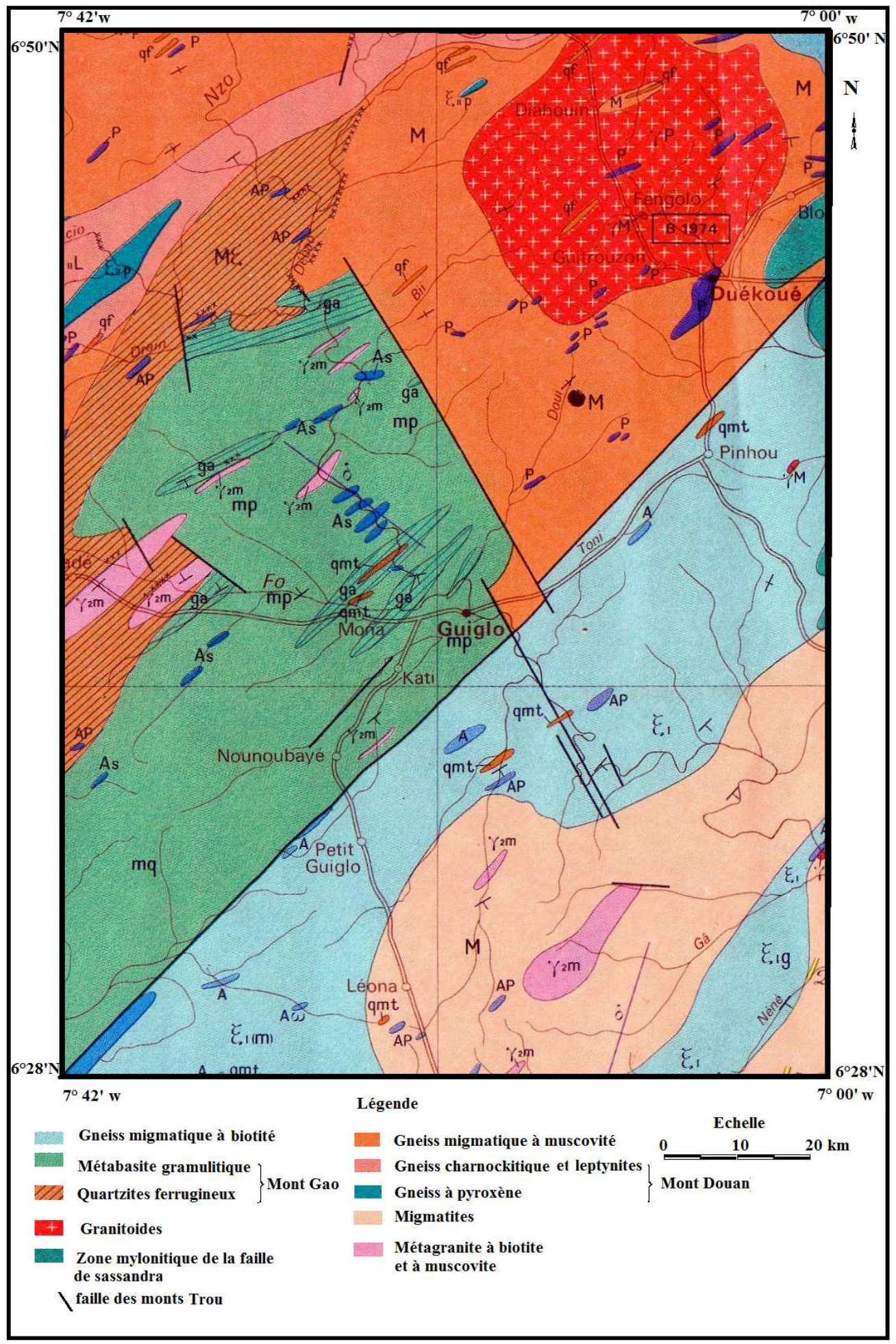

Figure 2: Carte géologique de la zone Guiglo-Duekoué (Ouest de la Côte d'Ivoire). 
$\mathrm{Du}$ point de vue géologique, le secteur Guiglo-Duékoué est à cheval sur le domaine Archéen (Guiglo) et le domaine SassandraCavally (SASCA) (Duekoué). Selon Kouamé (2011), la pétrographie des formations géologiques de cette zone est marquée par trois unités principales que sont (Figure 2): l'unité des monts Gao, l'unité des monts Douan et l'unité des migmatites à biotite et microcline. L'unité des monts Gao est constituée de méta-sédiments et de granitoïdes associées ou non à des quartzites ferrugineux. L'unité des monts Douan est composée de gneiss charnockitique et leptynitiques et de gneiss à pyroxène. Elle est aussi composée de charnockites (roche magmatique composée de quartz, de microclines, de plagioclases et de feldspaths alcalins). La disposition des faciès pétrographiques au sein de cette unité suggère une organisation en antiforme avec au cœur, les charnockites et les gneiss leptynitiques sur les flancs. L'unité des migmatites à biotite et microcline est un ensemble migmatitique qui témoigne d'un important épisode de fusion anatectique des unités pétrographiques précitée. L'unité est composée principalement de quartz, de microcline, d'oligoclase, de la biotite, d'autres minéraux accessoires comme le grenat, et la muscovite. Les formations de la zone d'étude présentent une orientation préférentielle NNE-SSW due aux phases de tectonique souple des deux (2) orogenèses (léonienne et libérienne) qui s'y sont manifestées. A ces phases, on associe le métamorphisme et la migmatisation des roches antécinématiques accompagnés du rubanement des migmatites, de la schistosité de l'ensemble schisteux et de la foliation des amphibolites. Outre ces phases de tectonique souple, une phase cassante a participé à la déformation des roches du secteur. Elle a occasionné la mise en place de filons de pegmatites, de dolérites plus ou moins importants, quelques rares filons de quartz et une granitisation considérable. La zone d'étude est très fracturée (Lasm, 2000 ; Kouamé, 2011), ce qui est favorable à l'infiltration des eaux qui vont contribuer à la recharge des nappes et à la modification de la minéralisation des eaux souterraines. La région Guiglo-Duekoué, située en zone de socle cristallin et cristallophyllien, est recouverte par des couches d'altérites d'épaisseurs plus ou moins importants, avec des affleurements de roches granitiques par endroits. Deux types d'aquifères y ont pris naissance au cours des temps géologiques. Il s'agit des aquifères d'altérites et des aquifères de fissures (Lasm, 2000).

\section{Données de l'étude}

La présente étude est essentiellement basée sur l'analyse des fiches techniques de 48 forages d'eau couvrant l'ensemble de la zone d'étude. Les paramètres physiques utilisés dans cette étude sont la température, le pH, la turbidité et la conductivité électrique. Ces paramètres ont été mesurés in situ. L'appareillage utilisé sur le terrain est constitué d'un $\mathrm{pH}$-mètre Star 4 pour la mesure du $\mathrm{pH}$, d'un conductimètre Hach Sension 5 pour la mesure de la conductivité électrique (CE) et de la température. La turbidité a été mesurée à l'aide d'un turbidimètre. En ce qui concerne les paramètres chimiques, cette étude s'est appuyée sur un certain nombre d'éléments chimiques qui sont le bicarbonate, le calcium, le magnésium, le sodium, le potassium, le chlore, le fer, le manganèse, le nitrate et le sulfate. Les échantillons d'eau destinés aux analyses chimiques ont été recueillis dans des bouteilles en polyéthylène pour être analysés par le service du Laboratoire d'Analyse d'Abengourou (LPA) en 2009. Ces analyses ont été réalisées à l'aide 
d'un spectrophotomètre DR 2010 de type HACH. Les données utilisées dans le cadre de cette étude proviennent du projet CESPPCC (Comité d'Examen et de Suivi des Projets et Programmes de la filière Café-Cacao) qui concerne la réalisation des forages d'exploitation équipés de pompes à motricité humaine dans l'Ouest de la Côte d'Ivoire. Elles sont issues de la consultation des fiches techniques de forages fournies par la Société Africaine de Forage (AFRIFOR).

\section{Analyse des faciès chimiques des eaux souterraines}

L'analyse hydrogéochimique a été réalisée à l'aide des digrammes de Piper et de Schoeller-Berkaloff. Le diagramme de Piper a été utilisé pour caractériser les faciès géochimiques des eaux de la zone GuigloDuekoué. Ce diagramme est très fréquemment utilisé et donne de très bons résultats (Yermani et al., 2003 ; Mohammad et Hamed, 2004 ; Tabouche et Achour, 2004 ; Alayat et Lamouroux, 2007 ; Kouzana et al., 2007 ; Oga et al., 2009 ; Kouassi et al., 2010 ; Yao et al., 2010). La détermination des formules hydrochimiques des faciès des eaux souterraines a été effectuée à partir du diagramme de Schoeller-Berkaloff qui est aussi beaucoup utilisé (Trabelsi et al., 2007 ; Yao et al., 2010). Les différents traitements ont été possibles grâce au logiciel DIAGRAMME ${ }^{\mathrm{TM}}$.

\section{Analyse des paramètres physico- chimiques des eaux souterraines}

La potabilité de l'eau est définie par des paramètres physiques, chimiques et biologiques, mais surtout en fonction de son usage. La potabilité de l'eau a été analysée au regard de l'usage humain et a concerné uniquement les paramètres physiques et chimiques. Une comparaison des teneurs en éléments physiques et chimiques des eaux des différents forages aux normes de l'Organisation Mondiale de la Santé (OMS, 2006) a été effectuée suivant Lallahem (2002). Les eaux seront jugées potables si les valeurs des paramètres physiques et chimiques définis plus haut sont dans les intervalles recommandés par l'Organisation Mondiale de la Santé (OMS, 2006).

Des études statistiques basées sur l'analyse des paramètres descriptifs de séries de valeurs ont été effectuées. Elles ont concerné les valeurs extrêmes (minimum, maximum), la moyenne (valeur centrale), l'écart-type (paramètre de dispersion) et le coefficient de variation $(\mathrm{Cv})$ qui se définit comme le rapport de l'écart-type à la moyenne. Selon la valeur de $\mathrm{Cv}$, trois structures de l'ensemble étudié sont mises en évidence :

- si $\mathrm{Cv}$ est inférieur à 0,20 , la série est très homogène ;

- si Cv est compris entre 0,20 et 0,25, la série est homogène ;

- si Cv est supérieur à 0,25 , la série est hétérogène.

Des paramètres physiques tels que la conductivité électrique et la dureté ont été en outre analysés à partir de critères de classification. La conductivité électrique des eaux a été classée selon le degré de minéralisation comme proposé par Rodier (1996) cité par Zourhi et Carlier (2002). Cette classification a été également utilisée par Ahoussi (2008), Soro (2010), Kouassi et al. (2010) :

- si $100<\mathrm{CE}<250 \mu \mathrm{S} / \mathrm{cm}$, eau faiblement minéralisée ;

- si $250<\mathrm{CE}<500 \mu \mathrm{S} / \mathrm{cm}$, eau peu minéralisée ;

- si $500<\mathrm{CE}<1000 \mu \mathrm{S} / \mathrm{cm}$, eau minéralisée ; - si $1000<\mathrm{CE}<3000 \mu \mathrm{S} / \mathrm{cm}$, eau fortement minéralisée. 
La classification d'une eau par rapport à la dureté donne les regroupements suivants (Ahoussi, 2008) :

- la classe des eaux douces pour une dureté inférieure à 15 degrés français ;

- la classe des eaux dures pour une dureté comprise entre 15 et 35 degrés français ;

- la classe des eaux très dures pour une dureté supérieure à 35 degrés français.

Les différents traitements liés aux caractéristiques descriptives et aux corrélations ont été réalisés à partir du tableur Microsoft Excel.

\section{RESULTATS}

\section{Hydrofaciès des eaux souterraines étudiées}

La projection des échantillons d'eau sur le diagramme de Piper a permis de mettre en évidence les différentes familles d'eau présentes dans la zone étudiée. Cette représentation met en évidence deux principales familles d'eau qui sont les eaux bicarbonatées calciques et magnésiennes et les eaux bicarbonatées sodi-potassiques (Figure 3). Les eaux bicarbonatées calciques et magnésiennes, faciès prédominant, représentent $95,83 \%$ des échantillons d'eau collectés dans la zone d'étude. En ce qui concerne les eaux bicarbonatées sodipotassiques $(4,17 \%)$, elles sont les moins représentées dans les eaux de la région de Guiglo-Duékoué.

Le diagramme de Schoeller-Berkaloff a permis de déterminer les différentes formules hydrochimiques liées aux différents hydrofaciès mis en évidence par le diagramme de Piper (Tableau 1).

\section{Résultats de l'analyse des paramètres physiques des eaux souterraines étudiées}

Les résultats de l'analyse des paramètres physiques sont consignés dans le Tableau 2. L'analyse de ce tableau révèle que les eaux souterraines de la région GuigloDuekoué ont des températures variant entre $25,7{ }^{\circ} \mathrm{C}$ et $28,9^{\circ} \mathrm{C}$ avec une moyenne de 26,7 ${ }^{\circ} \mathrm{C}$. Le pH de ces eaux est compris entre 6,51 et 7,13. Le pH moyen est de 6,67. La turbidité des eaux souterraines reste très faible dans l'ensemble. Les valeurs de turbidité varient entre 0,20 NTU et 1,01 NTU, avec une moyenne de 0,49 NTU. Elles sont en général inférieures ou égales à l'unité. Les eaux de la région contiennent peu de particules en suspension. La conductivité électrique des eaux varie entre 106 et $349 \mu \mathrm{s} / \mathrm{cm}$ avec une moyenne de 208,437 $\mu \mathrm{s} / \mathrm{cm}$. La majorité des échantillons d'eau a une conductivité électrique comprise entre $100 \mu \mathrm{s} / \mathrm{cm}$ et 250 $\mu \mathrm{s} / \mathrm{cm}(68,75 \%)$, classe correspondant aux eaux faiblement minéralisées contre 31,25\% correspondant aux eaux peu minéralisées $(250-350 \mu \mathrm{s} / \mathrm{cm})$. Les valeurs minimales et maximales de la dureté sont respectivement de $2,8^{\circ} \mathrm{F}$ et $15,40{ }^{\circ} \mathrm{F}$ avec une moyenne de 6,41 ${ }^{\circ} \mathrm{F}$. Les eaux souterraines de la zone DuekouéGuiglo sont donc douces. Les valeurs du coefficient de variation $(\mathrm{CV})$ des paramètres physiques sont très faibles $(0,02$ à 0,04$)$ pour la température, le $\mathrm{pH}$ et la turbidité alors qu'elles sont élevées pour la conductivité électrique $(0,33)$ et le TH total $(0,51)$. Ces résultats montrent que la température, le $\mathrm{pH}$ et la turbidité sont très homogènes donc varient très peu d'un point à un autre alors que la conductivité électrique et le $\mathrm{TH}$ total sont hétérogènes donc varient beaucoup dans l'espace.

\section{Résultats de l'analyse des paramètres chimiques des eaux souterraines étudiées}

Les résultats de l'analyse des ions majeurs sont consignés dans le Tableau 3. Les concentrations des ions majeurs varient entre $0 \mathrm{mg} \cdot \mathrm{L}^{-1}\left(\mathrm{NO}_{3}{ }^{-}\right.$et $\left.\mathrm{SO}_{4}{ }^{2-}\right)$ et $259 \mathrm{mg} \cdot \mathrm{L}^{-1}$ $\left(\mathrm{HCO}_{3}{ }^{-}\right)$. Les eaux de la zone Guiglo-Duekoué 


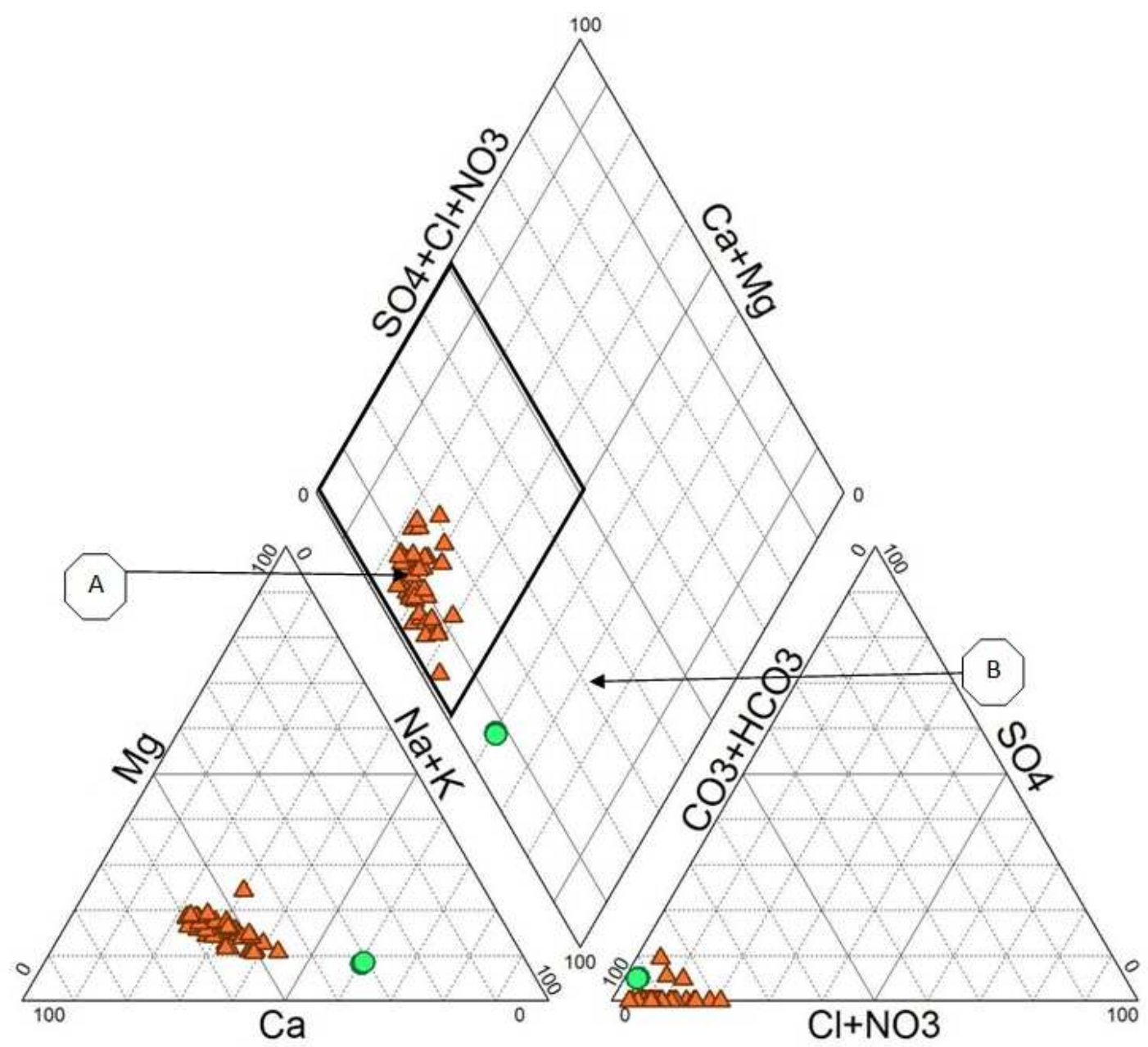

A : Eaux bicarbonatées calciques et magnésiennes

B : Eaux bicarbonatées sodi-potassiques

Figure 3: Classification hydrogéochimique des eaux souterraines à partir du diagramme de Piper. 
Tableau 1: Classification des faciès chimiques des eaux à partir du diagramme de SchoellerBerkaloff.

\begin{tabular}{llll}
\hline Formules & Hydrofaciès & Echantillons & Observations \\
\hline $\mathrm{rHCO}_{3}{ }^{-}>\mathrm{rSO}_{4}{ }^{2-}>\mathrm{rCl}^{-}$et & Eaux & $1 ; 2 ; 3 ; 4 ; 5 ; 6 ; 8 ; 10 ; 11 ; 12 ;$ & \\
$\mathrm{r}\left(\mathrm{Na}^{+}+\mathrm{K}^{+}\right)>\mathrm{rCa}^{2+}>\mathrm{rMg}^{2+}$ & bicarbonatées & $13 ; 14 ; 15 ; 16 ; 17 ; 20 ; 21 ; 22 ; 23 ;$ & \\
$\quad$ ou & calciques et & $24 ; 25 ; 26 ; 27 ; 28 ; 29 ; 30 ; 31 ; 32 ;$ & \\
$\mathrm{rHCO}_{3}^{-}>\mathrm{rCl}^{-}>\mathrm{rSO}_{4}{ }^{2-}$ et & magnésiennes & $33 ; 34 ; 35 ; 36 ; 37 ; 38 ; 39 ; 40 ; 41 ;$ & $95,83 \%$ \\
$\left.\mathrm{rCa}^{2+}>\mathrm{r}^{2} \mathrm{Na}^{+}+\mathrm{K}^{+}\right)>\mathrm{rMg}^{2+}$ & & $42 ; 43 ; 44 ; 45 ; 46 ; 47 ; 48$ & \\
\hline $\mathrm{rHCO}_{3}^{-}>\mathrm{rCl}^{-}>\mathrm{rSO}_{4}{ }^{2-}$ et & Eaux & & $4,17 \%$ \\
$\mathrm{r}\left(\mathrm{Na}^{+}+\mathrm{K}^{+}\right)>\mathrm{rCa}^{2+}>\mathrm{rMg}^{2+}$ & bicarbonatées & $18 ; 19 ;$ & \\
& sodi-potassiques & & \\
\hline
\end{tabular}

Tableau 2: Résultats d'analyse des paramètres physiques des eaux souterraines.

\begin{tabular}{lcccccc}
\hline Paramètres & Min & Max & Moy & Ecart-type & Cv & Norme OMS \\
\hline Température $\left({ }^{\circ} \mathrm{C}\right)$ & 25,7 & 28,9 & 26,7 & 0,55 & 0,02 & $25-30$ \\
pH & 6,51 & 7,13 & 6,67 & 0,13 & 0,02 & $6,5-8,5$ \\
Turbidité $(\mathrm{NTU})$ & 0,20 & 1,01 & 0,49 & 0,049 & 0,04 & $1-5$ \\
Conductivité $(\mu \mathrm{S} / \mathrm{cm})$ & 106 & 349 & 208,437 & 69,98 & 0,33 & 500 \\
TH total $\left({ }^{\circ} \mathrm{F}\right)$ & 2,8 & 15,4 & 6,41 & 3,3 & 0,51 & 30 \\
\hline
\end{tabular}

Tableau 3: Résultats d'analyse des paramètres chimiques des eaux souterraines.

\begin{tabular}{lcccccc}
\hline Paramètres & Min & Max & Moy & Ecart-type & $\mathbf{C v}$ & Norme OMS \\
\hline $\mathrm{Ca}^{2+}(\mathrm{mg} / \mathrm{l})$ & 8,82 & 47,2 & 19,36 & 10,23 & 0,52 & 100 \\
$\mathrm{Mg}^{2+}(\mathrm{mg} / \mathrm{l})$ & 1,94 & 8,75 & 3,35 & 2,02 & 0,60 & 50 \\
$\mathrm{Na}^{+}(\mathrm{mg} / \mathrm{l})$ & 3,94 & 17,42 & 10,37 & 4,17 & 0,31 & 150 \\
$\mathrm{~K}^{+}(\mathrm{mg} / \mathrm{l})$ & 1,43 & 6,33 & 3,77 & 1,20 & 0,46 & 10 \\
$\mathrm{HCO}_{3}{ }^{-}(\mathrm{mg} / \mathrm{l})$ & 61 & 259 & 154,52 & 60,91 & 0,39 & - \\
$\mathrm{Cl}^{-}(\mathrm{mg} / \mathrm{l})$ & 3,35 & 24,8 & 8,41 & 5,24 & 0,62 & 250 \\
$\mathrm{NO}_{3}{ }^{-}(\mathrm{mg} / \mathrm{l})$ & 0 & 05 & 0,23 & 0,77 & 0,62 & 50 \\
$\mathrm{SO}_{4}{ }^{2-}(\mathrm{mg} / \mathrm{l})$ & 0 & 10 & 1,25 & 3,34 & 2,66 & 250 \\
\hline
\end{tabular}


sont caractérisées par une prédominance des ions bicarbonates $(76,78 \%)$ sur les ions chlorures et sulfates qui sont en faible proportion dans ces eaux. Le calcium constitue le cation le plus important, puis viennent ensuite $\mathrm{Na}^{+}$et $\mathrm{K}^{+}$. Les valeurs du coefficient de variation (CV) des ions majeurs varient généralement $(87,5 \%)$ entre 0,31 et 0,62 . Cependant, au niveau des sulfates, on enregistre une valeur très élevée (supérieure à 1) qui est de 2,66. Ces résultats traduisent une grande hétérogénéité des ions majeurs. Les différentes concentrations des ions majeurs sont conformes aux normes de l'OMS.

Le fer et le manganèse ont des concentrations qui varie respectivement entre 0 et $0,66 \mathrm{mg} . \mathrm{L}^{-}$ ${ }^{1}$ pour le $\mathrm{Fe}^{2+} ; 0$ et $0,16 \mathrm{mg} . \mathrm{L}^{-1}$ pour le $\mathrm{Mn}^{2+}$. Le fer a une concentration moyenne de 0,15 $\mathrm{mg} . \mathrm{L}^{-1}$ alors que celle du manganèse est de $0,02 \mathrm{mg} . \mathrm{L}^{-1}$. Les valeurs du coefficient de variation $(\mathrm{CV})$ sont de 1,71 pour le fer et 0,80 pour le manganèse, ce qui exprime une forte hétérogénéité des ions métalliques. En ce qui concerne le fer, $85,42 \%$ de l'effectif des points d'eau ont des teneurs comprises entre 0 et $0,03 \mathrm{mg} \cdot \mathrm{L}^{-1}$. Ces valeurs sont inférieures à la norme de potabilité définie contre, 14,58\% qui ont des concentrations supérieures à la norme des eaux potables. Au niveau du manganèse, $77,09 \%$ des échantillons ont des concentrations comprises entre 0 et 0,05 $\mathrm{mg} \cdot \mathrm{L}^{-1}$. Ces concentrations sont inférieures à la norme de potabilité définie contre $22,91 \%$ dont les concentrations excèdent la norme.

\section{DISCUSSION}

L'étude hydrochimique a permis de mettre en évidence la prédominance de faciès bicarbonaté dans les eaux souterraines de la zone Guiglo-Duékoué. Ce résultat est en accord avec celui de plusieurs auteurs ayant travaillé en Afrique de l'Ouest et qui ont tous abouti à la conclusion selon laquelle, le faciès bicarbonaté est le plus représenté dans les eaux du socle (Soro, 2002 ; Ahoussi, 2008 ; Oga et al., 2009 ; Kouassi et al., 2010 ; Soro, 2010 ; Yao et al., 2010 ; Lasm et al., 2011). La dominance du faciès bicarbonaté dans les eaux de socle est à rattacher à l'origine de la production des ions bicarbonatés. En effet, les bicarbonates sont essentiellement produits par l'altération des silicates lors de l'acquisition de la charge saline des eaux dans la zone d'aération. La prédominance $\mathrm{du}$ faciès bicarbonaté confère un caractère homogène aux eaux de la zone d'étude. Cette homogénéité est liée à la faible hétérogénéité lithologique des roches de la région (Kouassi et al., 2010). En effet, la géologie de la région est largement dominée par les formations migmatitiques sur les autres formations géologiques (Gronayes et al., 2003). L'abondance des ions bicarbonates dans ces eaux peut s'expliquer par l'hydrolyse des feldspaths, constituant principal des gneiss charnockitiques et leptynitiques. Les cations dominants qui s'associent à ces ions

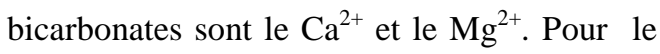
calcium, ils proviennent des formations géologiques qui forment les aquifères, particulièrement les roches volcaniques basiques métamorphisées (métabasalte, métaandésite, métadolérite, métagabbro, amphibolite) (Faillat et blavoux, 1989). La prédominance des ions $\mathrm{Na}^{+}$sur les ions $\mathrm{K}^{+}$ dans les eaux souterraines de la région de Guiglo-Duékoué s'explique d'une part par la grande stabilité de la muscovite et des feldspaths potassiques et d'autre part par l'adsorption des ions potassium par les plantes (Oga et al., 2009) sachant que la zone est fortement boisée. Les ions $\mathrm{K}^{+}$peuvent également être adsorbés par les minéraux néoformés.

Les températures des eaux souterraines étudiées varient entre $25,90{ }^{\circ} \mathrm{C}$ et $28,90{ }^{\circ} \mathrm{C}$. 
La norme étant fixée de 25 à $30^{\circ} \mathrm{C}$. Ces eaux sont conformes à la recommandation de l'OMS. Les eaux sont relativement acides à l'exception du forage du village de «Trois Maisons » qui a un pH égal à 7,13. Cette acidité est principalement liée à la production de $\mathrm{CO}_{2}$ sous l'action des activités biologiques (Lasm et al., 2008). La réaction d'hydratation du $\mathrm{CO}_{2}$ libère de l'acide carbonique qui attaque les roches (Goné et al., 2004). La valeur moyenne $(6,67) \mathrm{du} \mathrm{pH}$ mesurée est légèrement supérieure à celles enregistrées dans les forages du bassin de la Haute Marahoué ( $\mathrm{pH}=6,37$ ) par Biemi (1992), dans le degré carré de Grand-Lahou $(6,08)$ par Soro (2002), dans la région d'Abidjan (5,04 à 5,26) par Oga (1998), dans la région du N'ziComoé (6,58) par Kouassi et al. (2010), dans la région des lacs $(5,86)$ par Soro (2010). Mais, ce $\mathrm{pH}$ moyen est relativement inferieure à la moyenne trouvé dans le bassin de la Mé $(6,87)$ par Soro (1987). En général, les eaux naturelles ont des valeurs de $\mathrm{pH}$ qui sont comprises entre 4,5 et 9 (Kortatsi, 2007). Les valeurs de $\mathrm{pH}$ de la zone Guiglo-Duekoué sont en harmonie avec le $\mathrm{pH}$ des eaux souterraine de la Côte d'Ivoire. Les valeurs de la turbidité varient autour de 0,49 \pm 0,95 NTU. Ces eaux contiennent peu de particules en suspension. La dureté des eaux est très faible avec une moyenne de $6,41^{\circ} \mathrm{F}$. Les eaux de la zone Guiglo-Duékoué sont douces et respectent la norme de l'OMS (2006). Les valeurs de conductivité électrique des eaux sont conformes aux normes de l'OMS.

La concentration maximale de fer acceptable dans une eau de boisson a été fixée à $0,3 \mathrm{mg} \cdot \mathrm{L}^{-1}$ par l'OMS (2006). Cette norme a été définie pour répondre plus à des critères esthétique et organoleptique qu'à des critères sanitaires. En effet, l'ingestion de fer semble plus nécessaire à l'homme. Cet élément est généralement conseillé aux hommes qui ont une carence en fer. Tout comme le fer, une concentration de manganèse est gênante pour l'organisme de l'homme dans une certaine proportion. Ces deux éléments sont souvent rencontrés ensemble dans la nature sous une forme bivalente. Les fortes teneurs en fer et en manganèse enregistrées dans certaines localités constituent un problème majeur. En effet, ces deux éléments confèrent à l'eau un goût métallique désagréable, un aspect et une couleur rouge brun et marron noir respectivement pour le fer et pour le manganèse, qui amènent les populations rurales à se tourner vers d'autres sources d'approvisionnement dont les qualités bactériologiques et parasitologiques sont douteuses. Ce qui peut avoir des conséquences graves sur la santé des populations. Un développement de bactéries ferrugineuses augmentant la corrosion peut être observé dans les forages, les rendant vulnérables à d'autres sources de pollution qui dégraderaient considérablement la qualité de ces eaux (Oga et al., 2009). En outre, le fer peut être en solution vraie, à l'état colloïdal, plus ou moins combiné à la matière organique, sous forme de complexes organiques ou minéraux ou sous forme de particules en suspension. Au contact de l'air, l'ion ferreux $\mathrm{Fe}^{2+}$ (soluble) est oxydé en ion ferrique $\mathrm{Fe}^{3+}$ (insoluble). Ainsi, la présence de l'oxyde de fer contenu dans l'eau confère à celle-ci sa turbidité et sa couleur rouge (Lasm et al., 2008). La zone d'étude est relativement arrosée, avec une végétation capable de produire une bonne quantité de matière organique. Le fer et le manganèse semblent aussi provenir de l'altération des roches de surface (Lasm et al., 2008). La source du fer et du manganèse serait liée à la décomposition des minéraux ferromagnésiens tels que la biotite et l'amphibole (Kouassi et al., 2010). Cette hypothèse est confirmée par la géologie 
de notre milieu d'étude. Les formations géologiques de la zone Guiglo-Duekoué sont constituées de roches métamorphiques contenant de l'amphibole (gneiss charnockitiques leptynitiques) et riches en biotite (migmatite à biotite). L'altération de ces roches libère entre autres les $\mathrm{Fe}^{2+}$ qui sont par la suite entraînées vers les eaux souterraines. La région étant abondamment arrosée, le pluviolessivage des couches superficielles entraîne un départ important de plusieurs cations dont le $\mathrm{Mn}^{2+}$ vers les nappes. Une autre source du fer dans les eaux souterraines pourrait être le lessivage des silicates ferromagnésiens (biotite et pyroxènes). De même, il est fort probable que le fer provienne de la dissolution des roches migmatiques souvent associée à l'hématite en présence de matières organiques (Kortatsi, 2007 ; Kortatsi et al., 2008). Les fortes teneurs en fer et en manganèse des eaux souterraines de la région proviendraient essentiellement des terrains géologiques. Ainsi, selon les travaux de Gronayes et al. (2003) les fortes teneurs fer et en manganèse observées dans la région Guiglo-Duekoué sont liées à la présence des minéraux ferromagnésiens tels que les pyroxènes, les amphiboles et les biotites dans cette region. Ces fortes teneurs peuvent être atténuées par le traitement au charbon actif, ce qui permettra de supprimer la coloration des eaux.

\section{Conclusion}

L'étude hydrogéochimique réalisée sur les aquifères fissurés de la zone GuigloDuekoué a montré que les eaux souterraines se regroupent en deux principaux hydrofaciès qui sont les eaux bicarbonatées calciques et magnésiennes $(95,83 \%)$ et les eaux bicarbonatées sodi-potassiques $(4,17 \%)$. Ces eaux ont des températures qui varient entre $25,7^{\circ} \mathrm{C}$ et $28,9^{\circ} \mathrm{C}$ avec une moyenne de 26,7
${ }^{\circ} \mathrm{C}$. Leur $\mathrm{pH}$ est compris généralement entre 6,51 et 7,13. La majorité des eaux a un $\mathrm{pH}$ moyen égal à 6,58 , ce qui est proche de 7 . La turbidité des eaux souterraines reste très faible dans l'ensemble. De même, les valeurs des mesures varient entre $0,20 \mathrm{NTU}$ et 1,01 NTU, avec une moyenne de 0,49 NTU. Les eaux souterraines de la région contiennent donc peu de particules en suspension. La conductivité électrique des eaux varie entre 106 et 349 $\mu \mathrm{S} / \mathrm{cm}$, avec une moyenne de 208,437 $\mu \mathrm{S} / \mathrm{cm}$. En effet, la majorité des échantillons d'eau a une conductivité électrique comprise entre $100 \mu \mathrm{S} / \mathrm{cm}$ et $250 \mu \mathrm{S} / \mathrm{cm}(68,75 \%)$, classe correspondant aux eaux faiblement minéralisées contre $31,25 \%$ correspondant aux eaux peu minéralisées. La dureté des eaux varie entre $2,8{ }^{\circ} \mathrm{F}$ et $15,40{ }^{\circ} \mathrm{F}$, avec une moyenne de $6,41{ }^{\circ} \mathrm{F}$. Au niveau des ions majeurs, les concentrations moyennes varient entre $0 \mathrm{mg} \cdot \mathrm{L}^{-1}\left(\mathrm{NO}_{3}{ }^{-}\right)$et $259 \mathrm{mg} \cdot \mathrm{L}^{-1}\left(\mathrm{HCO}_{3}{ }^{-}\right)$. Les bicarbonates se révèlent comme les ions prédominants (76,78\%). L'étude des paramètres physico-chimiques a montré la grande variabilité chimique des eaux souterraines. Cette étude a révélé également que les eaux souterraines sont physicochimiquement conformes dans l'ensemble aux normes recommandées par l'OMS et ne présentent pas de danger majeur pour la consommation humaine. Cependant, il existe de fortes teneurs en fer et en manganèse dans les eaux, ce qui explique l'apparence rougeâtre et le goût désagréable de ces eaux. Dans ces conditions, les populations rurales ont recours à d'autres sources d'approvisionnement dont les qualités bactériologiques et parasitologiques sont douteuses. Ces anomalies pourraient être corrigées en traitant ces eaux avec le charbon actif. 


\section{REMERCIEMENTS}

Les auteurs de cet article remercient la Société Africaine de Forage (AFRIFOR) pour leur avoir fourni les données d'analyses physico-chimiques utilisées dans cette étude. Ils remercient également les instructeurs dont les critiques et les suggestions ont permis d'améliorer le présent article.

\section{REFERENCES}

Ahoussi KE. 2008. Evaluation quantitative et qualitative des ressources en eau dans le Sud de la Côte d'Ivoire. Application de l'hydrochimie et des isotopes de l'environnement à l'étude des aquifères continus et discontinus de la région d'Abidjan-Agboville. Thèse de Doctorat de l'Université de Cocody-Abidjan, Côte d'Ivoire, p.270.

Alayat H, Lamouroux C. 2007. Caractérisation physico-chimique des eaux thermo-minérales des monts de la Cheffia (extrême Nord-Est algérien). La Presse thermale et climatique, 144: 191199.

Biémi J. 1992. Contribution à l'étude géologique, hydrogéologique et par télédétection des bassins versants Subsahéliens du socle précambrien d'Afrique de l'Ouest : Hydrostructurale, hydrochimie et isotopie des aquifères discontinus des sillons et aires granitiques de la Haute Marahoué (Côte d'Ivoire). Thèse de Doctorat d'Etat es Sciences Naturelle, Université Nationale de Côte d'Ivoire, p. 480.

Brou YT. 2005. Climat, mutations socioéconomiques et paysages en Côte d'Ivoire. Mémoire de synthèse des activités scientifiques présenté en vue de l'obtention de l'Habilitation à Diriger des Recherches, Université des sciences et technologies de Lille, France, p.226.
Faillat JP, Blavoux B. 1989. Caractères hydrochimiques des nappes des roches endogènes fissurées en zone tropicale humide : l'exemple de la Côte d'Ivoire. $J$. Afri. Earth. Sc., 9(1): 31- 40.

Gronayes CC, Koffi PB, Kouamé KF, Kouakou BB. 2003. Identification de couloirs de cisaillement à partir de l'imagerie TM de landsat : Application à l'Archéen de Bloléquin (Ouest de la Côte d'Ivoire). Bioterre, 2: 218-221.

Kouamé KF. 2011. Gestion intégrée des ressources en eau en Afrique tropicale humide. Cas du bassin versant du N'zo à l'Ouest de la Côte d'Ivoire. Contribution de la télédétection, des systèmes d'informations géographiques et de la modélisation hydrologique. Thèse de Doctorat d'État, Université de CocodyAbidjan, Côte d'Ivoire, p.250.

Kouassi AM, Yao KA, Ahoussi KE, Seki CL, Yao NA, Kouassi KI. 2010. Apports des méthodes statistiques et hydrochimiques à la caractérisation des eaux des aquifères fissurés de la région du N'zi-Comoé (Centre-Est de la Côte d'Ivoire). International Journal of Biological and Chemical Sciences, 4(5): 1816-1838.

Kouzana L, Ben Mammou A, Gaaloul N. 2007. Intrusion marine et salinisation des eaux d'une nappe phréatique côtière (Korba, Cap-Bon, Tunisie). Geo-EcoTrop., 31: 57-70.

Kortatsi BK. 2007. Hydrochemical framework of groundwater in the Ankobra Basin, Ghana. Aquat. Geochem., 13: 41-74.

Kortatsi BK, Tay CK, Anornu G, Hayford E, Dartey GA. 2008. Hydrogeochemical evaluation of groundwater in the lower Offin basin, Ghana. Environ. Geol., 53 (8): 1651-1662. 
Lalbat F. 2006. Fonctionnement hydrodynamique de l'aquifère du Miocène $\mathrm{du}$ bassin de Carpentras (Vaucluse, France). Thèse de Doctorat, Université d'Avignon et des pays de Vaucluse, France, p.234.

Lallahem S. 2002. Structure et modélisation hydrodynamique des eaux souterraines : application à l'aquifère crayeux de la bordure nord du bassin de paris. Thèse de Doctorat, Université de Lille, France, p.243.

Lasm T. 2000. Hydrogéologie des réservoirs fracturés de socle : Analyses statistique et géostatistique de la fracturation et des propriétés hydrauliques. Application à la région des montages de Côte d'Ivoire (domaine archéen). Thèse de l'Université de Poitiers, p.274.

Lasm T, Yao KT, Oga MS, Kouame KF, Jourda P, Kouadio KE, Baka D. 2008. Analysis of the physico-chemical characteristics of groundwater in proterozoic land region of the Tiassale area (Southern Côte d'Ivoire). European Journal of Scientific Research, 20(3): 526-543.

Lasm T, ASM, Lasme O, Oga MS, Youanta M, Baka D, Kouamé KF, Yao KT. 2011. Caractérisation hydrochimique des aquifères fissurés de la région de SanPedro (Sud-Ouest de la Côte d'Ivoire). International Journal of Biological and Chemical Sciences, 5(2): 642-662.

Mohammad RAA, Hamed AEN. 2004. Hydrochemical facies of groundwater in the Gaza Strip, Palestine. Journal des Sciences Hydrologiques, 49(3): 359-371.

Oga MS. 1998. Ressources en eaux souterraines dans la région du Grand Abidjan (Côte d'Ivoire): Approche Hydrochimique et Isotopique. Thèse de
Doctorat, Université de Paris Orsay, p.240.

Oga MS, Lasm T, Yao KT, Soro N, Saley MB, Kouassi D, Gnamba F. 2009. Caractérisation chimique des eaux des aquifères de Fracture: cas de la région de Tiassalé en Cote D'ivoire. European Journal of Scientific Research, 31 (1): 7287.

O.M.S. 2006. Normes de l'OMS sur l'eau potable. O.M.S.

Soro N. 2002. Hydrochimie et géochimie isotopique des eaux souterraines du degré carré de Grand-Lahou et ses environs (Sud-Ouest de la Côte d'Ivoire). Implication hydrologique et hydrogéologique. Thèse de Doctorat ès sciences naturelles, Université de Cocody-Abidjan, Côte d'Ivoire, p.272.

Soro G. 2010. Évaluation quantitative et qualitative des ressources en eaux souterraines dans la région des lacs (centre de la côte d'ivoire) : Hydrogéologie et hydrochimie des aquifères discontinus du district de Yamoussoukro et du département de Tiebissou. Thèse de Doctorat de l'Université de Cocody-Abidjan, Côte d'Ivoire, p. 257.

Tabouche N, Achour S. 2004. Etude de la qualité des eaux souterraines de la région orientale du Sahara septentrional algérien. Larhyss Journal, 3: 99-113.

Trabelsi S, Bouri S, Ben Dhia H. 2007. Contribution des approches hydrochimiques et géothermométriques à l'étude des eaux thermales de la province fluorée tunisienne (Nord-Est Tunisien). Revue des Energies Renouvelables, 7: 8588.

Yao KA, Kouassi AM, Koffi YB, Biemi J. 2010. Caractérisation hydrodynamique et hydrogéochimique des aquifères fissurés 
de la région de Toumodi (Centre de la Côte d'Ivoire). Journal of Environmental Hydrology, 18 (26): 1-15.

Yermani M, Zouari K, Michelot JL, Mamou A, Moumni L. 2003. Approche géochimique du fonctionnement de la nappe profonde de Gafsa Nord (Tunisie centrale). Journal des Sciences Hydrologiques, 48(1): 95-108.

Zourhi L, Carlier E. 2002. Caractérisation hydrochimique d'une nappe côtière, Maroc. Journal of environment hydrology, 9(4): 1-7. 